\title{
Remarks on a Paper by Ahmad, Ahmad and Ahmed
}

G.G. Hamedani

Department of Mathematics, Statistics and Computer Science

Marquette University, Milwaukee, WI 53201-1881

g.hamedani@mu.edu

\begin{abstract}
Ahmad et al. (2015) consider a Transmuted Kumaraswamy distribution and study certain properties of their distribution. In the title of their paper they mention characterization of this distribution, but no characterizations are presented in the paper. In the present short note we establish certain characterizations of the Transmuted Kumaraswamy in three directions.
\end{abstract}

\section{Introduction}

Characterizations of distributions is an important research area which has recently attracted the attention of many researchers. This short note deals with various characterizations of Transmuted Kumaraswamy (TK) distribution to complete, in some way, the work of Ahmad et al. (2015). These characterizations are based on: (i) a simple relationship between two truncated moments; (ii) the hazard function; (iii) a single function of the random variable. It should be mentioned that for characterization $(i)$ the $c d f$ (cumulative distribution function) need not have a closed form. The main purpose of this short note is the presentation of mathematically elegant results in the field of characterization of distributions. We hope that the finding of this work will be helpful to the applied scientists.

Ahmad et al. (2015) introduced TK distribution with $c d f$ and $p d f$ (probability density function) given, respectively, by

$$
F(x ; \theta, \alpha, \lambda)=\left[1-\left(1-x^{\theta}\right)^{\alpha}\right]\left[1+\lambda\left(1-x^{\theta}\right)^{\alpha}\right]
$$

and

$$
f(x ; \theta, \alpha, \lambda)=\theta \alpha x^{\theta-1}\left(1-x^{\theta}\right)^{\alpha-1}\left[1-\lambda+2 \lambda\left(1-x^{\theta}\right)^{\alpha}\right],
$$

for $x \in(0,1)$, where $\theta, \alpha$ positive and $|\lambda| \leq 1$ are parameters.

\section{Characterizations of TK distribution}

We present our characterizations (i)-(iii) in three subsections.

\subsection{Characterizations based on two truncated moments}

In this subsection we present characterizations of TK distribution in terms of a simple relationship between two truncated moments. This characterization result employs a theorem due to Glänzel [2], see Theorem 2.1.1 below. Note that the result holds also when the interval $H$ is not closed. Moreover, as mentioned above, it could be also applied when the cdf $F$ does not have a closed form. As shown in [3], this characterization is stable in the sense of weak convergence. 
Theorem 2.1.1. Let $(\Omega, \mathcal{F}, \mathbf{P})$ be a given probability space and let $H=[d, e]$ be an interval for some $d<e \quad(d=-\infty, e=\infty$ mightaswellbeallowed). Let $X: \Omega \rightarrow H$ be a continuous random variable with the distribution function $F$ and let $g$ and $h$ be two real functions defined on $H$ such that

$$
\mathbf{E}[g(X) \mid X \geq x]=\mathbf{E}[h(X) \mid X \geq x] \xi(x), \quad x \in H,
$$

is defined with some real function $\eta$. Assume that $g, h \in C^{1}(H), \xi \in C^{2}(H)$ and $F$ is twice continuously differentiable and strictly monotone function on the set $H$. Finally, assume that the equation $\xi h=g$ has no real solution in the interior of $H$. Then $F$ is uniquely determined by the functions $g, h$ and $\xi$, particularly

$$
F(x)=\int_{a}^{x} C\left|\frac{\xi^{\prime}(u)}{\xi(u) h(u)-g(u)}\right| \exp (-s(u)) d u,
$$

where the function $s$ is a solution of the differential equation $s^{\prime}=\frac{\xi^{\prime} h}{\xi h-g}$ and $C$ is the normalization constant, such that $\int_{H} d F=1$.

Proposition 2.1.1. Let $X: \Omega \rightarrow(0,1)$ be a continuous random variable and let $h(x)=$ $\left[1-\lambda+2 \lambda\left(1-x^{\theta}\right)^{\alpha}\right]^{-1}$ and $g(x)=h(x)\left(1-x^{\theta}\right)$ for $x \in(0,1)$. The random variable $X$ belongs to TK family (1.2) if and only if the function $\xi$ defined in Theorem 2.1.1 has the form

$$
\xi(x)=\frac{\alpha}{\alpha+1}\left(1-x^{\theta}\right), \quad x \in(0,1) .
$$

Proof. Let $X$ be a random variable with pdf (1.2), then

$$
(1-F(x)) E[h(x) \mid X \geq x]=\left(1-x^{\theta}\right)^{\alpha}, \quad x \in(0,1),
$$

and

$$
(1-F(x)) E[g(x) \mid X \geq x]=\frac{\alpha}{\alpha+1}\left(1-x^{\theta}\right)^{\alpha+1}, \quad x \in(0,1),
$$

and finally

$$
\xi(x) h(x)-g(x)=-\frac{1}{\alpha+1} h(x)\left(1-x^{\theta}\right)<0 \quad \text { for } x \in(0,1) .
$$

Conversely, if $\xi$ is given as above, then

$$
s^{\prime}(x)=\frac{\xi^{\prime}(x) h(x)}{\xi(x) h(x)-g(x)}=\frac{\alpha \theta x^{\theta-1}}{\left(1-x^{\theta}\right)}, \quad x \in(0,1),
$$

and hence

$$
s(x)=-\log \left\{\left(1-x^{\theta}\right)^{\alpha}\right\}, \quad x \in(0,1) .
$$

Now, in view of Theorem 2.1.1, $X$ has density (1.2). 
Corollary 2.1.1. Let $X: \Omega \rightarrow(0,1)$ be a continuous random variable and let $h(x)$ be as in Proposition 2.1.1. The pdf of $X$ is (1.2) if and only if there exist functions $g$ and $\xi$ defined in Theorem 2.1.1 satisfying the differential equation

$$
\frac{\xi^{\prime}(x) h(x)}{\xi(x) h(x)-g(x)}=\frac{\alpha \theta x^{\theta-1}}{\left(1-x^{\theta}\right)}, \quad x \in(0,1) \text {. }
$$

The general solution of the differential equation in Corollary 2.1.1 is

$$
\xi(x)=\left(1-x^{\theta}\right)^{-\alpha}\left[-\int \alpha \theta x^{\theta-1}\left(1-x^{\theta}\right)^{\alpha-1}(h(x))^{-1} g(x)+D\right],
$$

where $D$ is a constant. Note that a set of functions satisfying the above differential equation is given in Proposition 2.1.1 with $D=0$. However, it should be also noted that there are other triplets $(h, g, \xi)$ satisfying the conditions of Theorem 2.1.1.

\subsection{Characterization based on hazard function}

It is known that the hazard function, $h_{F}$, of a twice differentiable distribution function, $F$, satisfies the first order differential equation

$$
\frac{f^{\prime}(x)}{f(x)}=\frac{h_{F}^{\prime}(x)}{h_{F}(x)}-h_{F}(x) \text {. }
$$

For many univariate continuous distributions, this is the only characterization available in terms of the hazard function. The following characterization establishes a non-trivial characterization for TK distribution in terms of the hazard function, which is not of the trivial form given in (2.2.1).

Proposition 2.2.1. Let $X: \Omega \rightarrow(0,1)$ be a continuous random variable. The pdf of $X$ is (1.2) if and only if its hazard function $h_{F}(x)$ satisfies the differential equation

$$
h_{F}^{\prime}(x)-(\theta-1) x^{-1} h_{F}(x)=\alpha \theta \frac{d}{d x}\left\{\frac{\left[1-\lambda+2 \lambda\left(1-x^{\theta}\right)^{\alpha}\right]}{\left(1-x^{\theta}\right)\left[1-\lambda+\lambda\left(1-x^{\theta}\right)^{\alpha}\right]}\right\} \text {. }
$$

Proof. If $X$ has pdf (1.2), then clearly (2.2.2) holds. Now, if (2.2.2) holds, then

$$
\frac{d}{d x}\left\{x^{-(\theta-1)} h_{F}(x)\right\}=\alpha \theta \frac{d}{d x}\left\{\frac{\left[1-\lambda+2 \lambda\left(1-x^{\theta}\right)^{\alpha}\right]}{\left(1-x^{\theta}\right)\left[1+\lambda\left(1-x^{\theta}\right)^{\alpha}\right]}\right\},
$$

or, equivalently,

$$
h_{F}(x)=\frac{\alpha \theta x^{\theta-1}\left[1-\lambda+2 \lambda\left(1-x^{\theta}\right)^{\alpha}\right]}{\left(1-x^{\theta}\right)\left[1-\lambda+\lambda\left(1-x^{\theta}\right)^{\alpha}\right]}
$$

which is the hazard function of the TK distribution. 


\subsection{Characterization based on truncated moment of certain function of the random variable}

The following propositions have already appeared in our unpublished (Hamedani, Technical Report, 2013) or previous work, so we will just state them here which can be used to characterize TK distribution.

Proposition 2.3.1. Let $X: \Omega \rightarrow(a, b)$ be a continuous random variable with cdf $F$. Let $\psi(x)$ be a differentiable function on $(a, b)$ with $\lim _{x \rightarrow a^{+}} \psi(x)=1$. Then for $\delta \neq 1$,

$$
E[\psi(X) \mid X \geq x]=\delta \psi(x), \quad x \in(a, b),
$$

if and only if

$$
\psi(x)=(1-F(x))^{\frac{1}{\delta}-1}, \quad x \in(a, b) .
$$

Proposition 2.3.2. Let $X: \Omega \rightarrow(a, b)$ be a continuous random variable with cdf $F$. Let $\psi_{1}(x)$ be a differentiable function on $(a, b)$ with $\lim _{x \rightarrow b^{-}} \psi_{1}(x)=1$. Then for $\delta_{1} \neq 1$

$$
E\left[\psi_{1}(X) \mid X \leq x\right]=\delta_{1} \psi_{1}(x), \quad x \in(a, b),
$$

if and only if

$$
\psi_{1}(x)=(F(x))^{\frac{1}{\delta_{1}}-1}, \quad x \in(a, b) .
$$

Remark 2.3.1. It is easy to see that for certain functions $\psi(x)$ and $\psi_{1}(x)$ on $(0,1) ;(a)$ Proposition 2.3.1 provides a characterization of TK distribution. (b) Proposition 2.3.2 provides a characterization of TK distribution as well.

\section{References}

1. Ahmad, A., Ahmad, S.P. and Ahmed, A. (2015). Characterization and estimation of transmuted Kumaraswamy distribution, Mathematical Theory and Modeling, 5 (9), 168-175.

2. Glänzel, W. (1987). A characterization theorem based on truncated moments and its application to some distribution families, Mathematical Statistics and Probability Theory (Bad Tatzmannsdorf, 1986), Vol. B, Reidel, Dordrecht, 75-84.

3. Glänzel, W. (1990). Some consequences of a characterization theorem based on truncated moments, Statistics: A Journal of Theoretical and Applied Statistics, 21 (4), 613-618. 